\title{
Optical Damage Threshold of Silicon for Ultrafast Infrared Pulses ${ }^{1}$
}

\author{
B. Cowan \\ Stanford Linear Accelerator Center, 2575 Sand Hill Road, Menlo Park, CA 94025
}

\begin{abstract}
While silicon has several properties making it an attractive material for structure-based laser-driven acceleration, its optical damage threshold, a key parameter for high-gradient acceleration, has been unknown. Here we present measurements of the optical damage threshold of crystalline silicon for ultrafast pulses in the mid-infrared. The wavelengths tested span a range from the telecommunications band at $1550 \mathrm{~nm}$ extending longer toward the two-photon absorption threshold at around $2200 \mathrm{~nm}$. We discuss the prevailing theories of ultrafast optical breakdown, describe the experimental setup and preliminary results, and propose a relevant performance parameter for candidate accelerator structures.
\end{abstract}

The following article has been submitted to 12th Advanced Accelerator Concepts Workshop (AAC 2006), Lake Geneva, WI, July 10-15, 2006. After it is published, it will be found at http://proceedings.aip.org/proceedings.

\section{INTRODUCTION}

Silicon is an attractive material for structure-based laser-driven acceleration for several reasons. It is transparent in the mid-infrared [1], in particular in the telecommunications band at $1550 \mathrm{~nm}$ where many promising sources exist (see for instance [2]). It has a high index of refraction at those wavelengths, which is generally required for the creation of photonic crystal structures with complete bandgaps [3]. It is resistant to radiation damage [4]. In addition, highly developed nanofabrication techniques exist for silicon due to its use in integrated circuits.

In previous work we have computationally demonstrated a speed-of-light accelerating mode in a structure based on a complete photonic bandgap material and investigated its properties, including particle beam dynamics and mode coupling [5]. However, the maximum sustainable accelerating gradient, a key parameter for an accelerator structure, has been unknown. This is because the optical damage threshold for potential materials, including silicon, has not previously been measured for wavelengths and laser pulse widths of interest. In the following section we will discuss the prevailing theories behind optical damage. Next we will describe an experiment to measure the optical breakdown threshold of crystalline silicon for ultrafast pulses in the infrared and present preliminary results. Finally, we will discuss a relevant performance parameter for accelerators and outstanding issues.

\footnotetext{
${ }^{1}$ Work supported by Department of Energy contracts DE-AC02-76SF00515 (SLAC) and DE-FG0397ER41043-II (LEAP).
} 


\section{THEORETICAL BACKGROUND}

As discussed in [6], optical breakdown in dielectric materials occurs in four general steps: (1) Seed conduction electrons are generated by multiphoton ionization (MPI) or tunnel ionization (TI), (2) they are accelerated in the laser field and generate an avalanche by impact ionization, (3) the laser pulse heats the resulting plasma, and (4) the electron energy is transferred to the lattice, resulting in ablation. This schematic description raises several questions. First, which process, MPI or TI, generates the seed electrons? Second, does the seed process or the avalanche dominate the free carrier generation? As a practical matter for accelerator structure design, can we significantly increase the damage threshold by lengthening the wavelength beyond a multiphoton threshold? For instance, the bandgap of silicon at room temperature is $1.12 \mathrm{eV}$, corresponding to a free-space wavelength of $1107 \mathrm{~nm}$. By using a wavelength longer than $1107 \mathrm{~nm}$, seed electrons can only be generated by two-photon absorption, which has a much lower cross-section than single-photon absorption. Similarly, operating at wavelengths beyond the two-photon threshold at $2214 \mathrm{~nm}$ might yield a further increase in sustainable gradient.

The experiments described in [6] were conducted at a wavelength of $800 \mathrm{~nm}$ on fused silica (bandgap energy $\Delta \approx 9 \mathrm{eV}$, requiring six-photon absorption) and barium aluminum borosilicate (BBS, $\Delta \approx 4 \mathrm{eV}$, requiring three-photon absorption). The authors reached the conclusion that multiphoton ionization dominates as the seed process for FWHM pulse widths $\tau \geq 20 \mathrm{fs}$, while tunneling dominates for shorter wavelengths. Further, they found that for fused silica, the avalanche dominates carrier generation for pulses as short as $10 \mathrm{fs}$, while for the lower-bandgap BBS, multiphoton ionization takes over for pulses shorter than $100 \mathrm{fs}$. Other experiments which have been conducted at $527 \mathrm{~nm}$ and $1053 \mathrm{~nm}$ are consistent with these conclusions [7]. However, another experiment measuring breakdown thresholds as a function of polarization has called into question the significance of MPI in the breakdown process [8]. Also, evidence indicates that TI is the dominant ionization process in the mid-infrared [9]. Therefore the sustainable gradient cannot be arbitrarily increased by using longer wavelengths.

\section{EXPERIMENTAL SETUP AND PROCEDURE}

As samples for the damage study, we used undoped crystalline silicon cut from a wafer with a (100) surface orientation. We conducted experiments starting at $\lambda=1550 \mathrm{~nm}$ and extending longer in wavelength toward $2200 \mathrm{~nm}$. The infrared pulses were generated by a commercial Spectra-Physics OPA-800 optical parametric amplifier (OPA) pumped by a Ti:sapphire laser system. The OPA produced pulses with energy $\geq 20 \mu \mathrm{J}$ and FWHM duration between 300 and $700 \mathrm{fs}$, depending on wavelength. The repetition rate of the laser was $240 \mathrm{~Hz}$, so the experiment detected multiple-shot damage.

This was a pump-probe measurement in which a CW helium-neon laser was focused on the same spot on the sample as the infrared pulses and damage was detected by observing a decrease in reflected HeNe intensity. A schematic of the experiment is shown in Figure 1. The infrared pulses were normally incident on the sample, focused by a $\mathrm{CaF}_{2}$ lens to minimize dispersion, while the HeNe beam was incident at an angle. The 


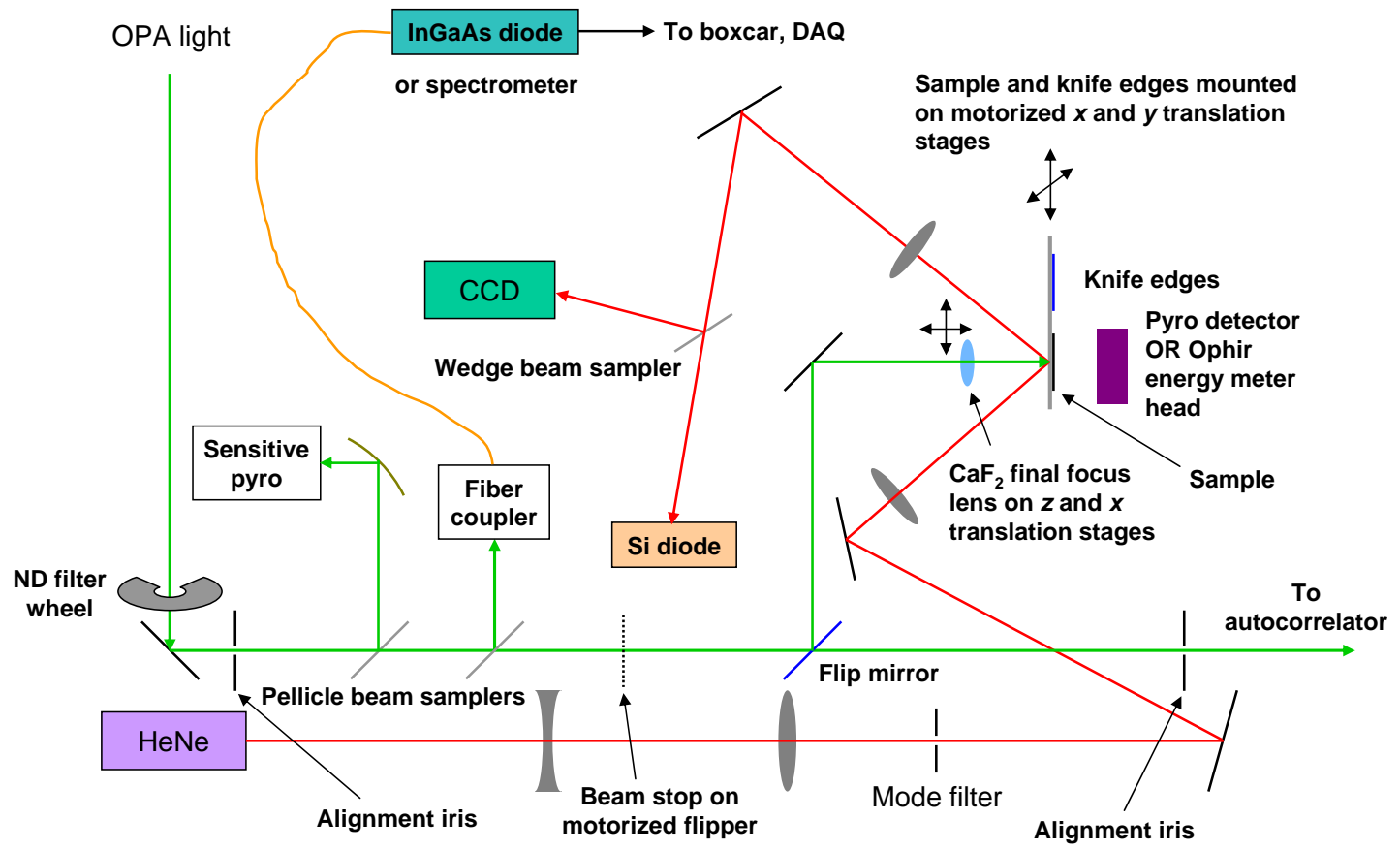

FIGURE 1. Schematic of the damage threshold measurement experiment.

sample was mounted vertically on motorized translation stages with motion in the plane of the sample. The sample was oriented during initial setup so that it was parallel to the directions of motion of the stages. Razor blades were mounted in the same plane as the sample to conduct knife-edge transverse spot size measurements. A photograph of the setup is shown in Figure 2.

For each wavelength tested, the infrared beam was first coupled via fiber to an optical spectrum analyzer (OSA) to confirm that no residual light at shorter wavelengths was present. Such light could have come from the Ti:sapphire pump at $800 \mathrm{~nm}$ or in the visible range from the pump mixed with the signal or the idler within the OPA. Next, an autocorrelation trace was taken to measure the pulse duration. Then, the final focus lens was adjusted to place the beam waist just in front of the sample surface. This was to ensure that maximum fluence occurred on the sample surface rather than in the bulk, so that the transverse spot-size measurements would yield relevant results. Also, the HeNe beam was spatially overlapped with the infrared pulses and one damage spot was created to confirm that pulse energy was sufficient and the focus was tight enough for damage to occur. Once this was complete, the transverse spot sizes were measured using the knifeedge technique. As a final setup step, the sensitive pyroelectric detector used to measure pulse energy was calibrated against an Ophir Optronics PE10 energy detector.

\section{RESULTS}

Once the setup was complete for a particular wavelength, damage data were taken. For each event, the pulses were allowed to illuminate the sample by removing a beam stop, 


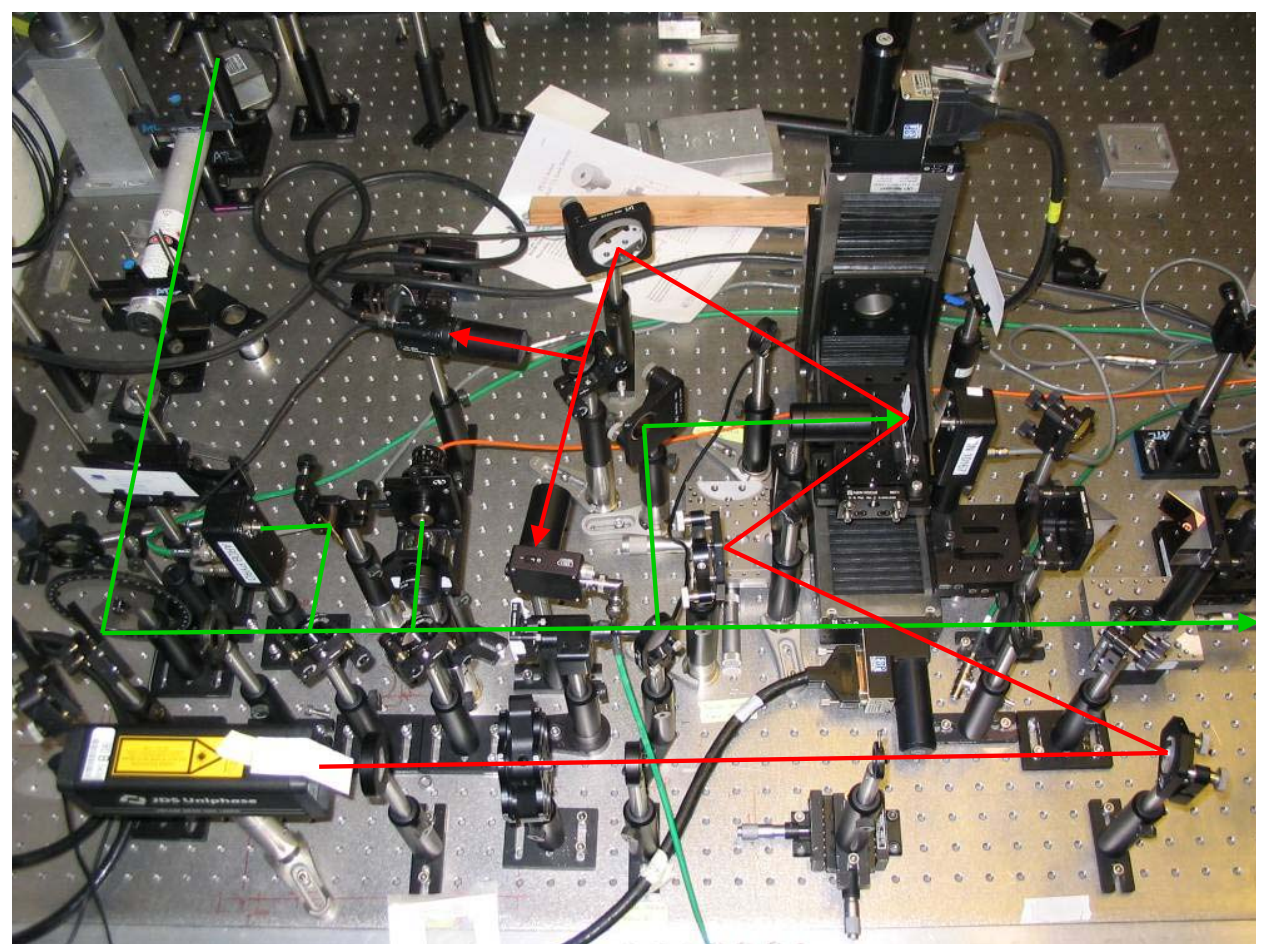

FIGURE 2. Photo of the damage threshold measurement experiment.

and infrared pulse energy and reflected HeNe power were then acquired on a shot-toshot basis. Since the data acquisition rate was limited to $\approx 100 \mathrm{~Hz}$, not all samples were acquired. The acquisition was stopped, and the beam stop reinserted, when either the HeNe power decreased, indicating damage, or a certain amount of time, usually $\geq 100 \mathrm{~s}$, had elapsed with no damage. Events were taken both above and below the damage threshold.

A sample event is plotted in Figure 3. We notice that the reflected HeNe power increases for a fraction of a second before falling off. This is ostensibly due to additional focusing from the silicon surface as the damage morphology develops. Indeed, we observed using a CCD image of the reflected HeNe light that the mode pattern changes for about a second during damage before finally disappearing.

From the multiple-shot data in this event and others like it, it is not immediately clear how to compute the measured damage threshold. We assume that damage was initiated by a single pulse, with further damage occurring in each subsequent shot due to the field enhancement resulting from the initial deformation of the surface. That pulse may or may not have been acquired, and the data do not tell us how long before the visible onset of damage the pulse occurred. However, we can make a maximum likelihood estimate for the damage threshold based on a few assumptions. First, the damage process is deterministic, as reported in [8], so damage did occur after a pulse that exceeded the threshold and did not otherwise. Second, at least one pulse with energy above threshold therefore must have occurred within the 1000 acquired shots before the visible onset of damage. Third, no such shot occurred more than 1000 shots before the visible onset 


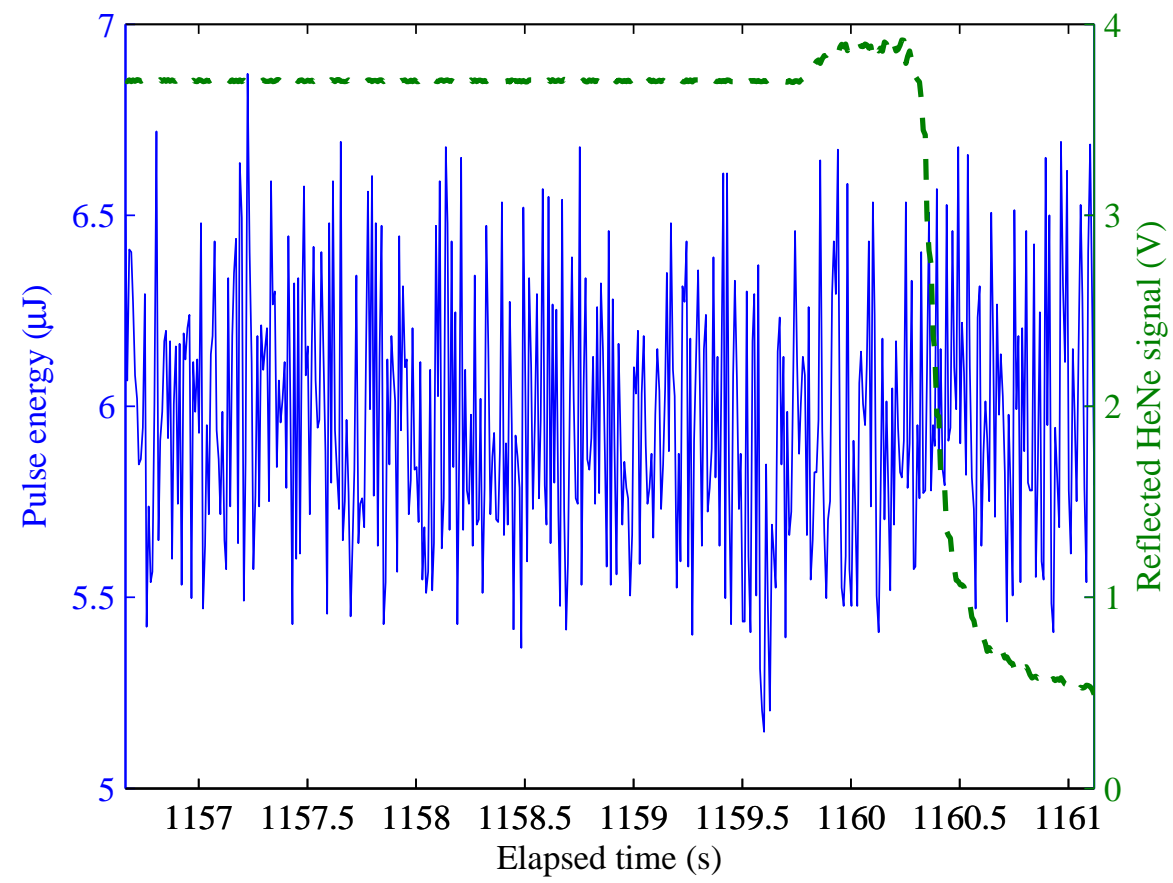

FIGURE 3. A sample event. Shown here are data from the last several seconds of an event in which damage occurred after about $1160 \mathrm{~s}$. The solid line shows a trace of the acquired pulse energy, and the dashed line shows the reflected HeNe power.

of damage, or more than 1000 shots before the end of an event in which no damage was observed. Forming a maximum likelihood estimate from these assumptions and the statistical parameters of the pulse energy distribution in an event, we can determine the damage threshold. For instance, for the run with the event shown, the threshold was $(7.78 \pm 0.05) \mu \mathrm{J}$.

We obtained full data sets for $\lambda=1550 \mathrm{~nm}, 1700 \mathrm{~nm}, 1900 \mathrm{~nm}$, and $2100 \mathrm{~nm}$. The preliminary damage threshold results are plotted in Figure 4. We notice that that the damage threshold does indeed increase as the wavelength approaches the two-photon absorption threshold, with the largest increase from $1700 \mathrm{~nm}$ to $1900 \mathrm{~nm}$. This is reasonable, since we would not expect a sharp cutoff from an MPI-dominated process because silicon has an indirect bandgap. However, the OPA pulse duration decreases, which might also account for the increase in threshold. One damage event was also taken at $2260 \mathrm{~nm}$, beyond the two-photon threshold, which set an upper bound on the damage threshold at $79 \mathrm{~J} / \mathrm{cm}^{3}$ for that wavelength.

\section{ACCELERATOR PERFORMANCE PARAMETER}

Note that in the description of the results we quoted damage thresholds in terms of energy density instead of fluence, which is the parameter conventionally used in optical damage literature. This is done both to reflect the possible importance of MPI in the 


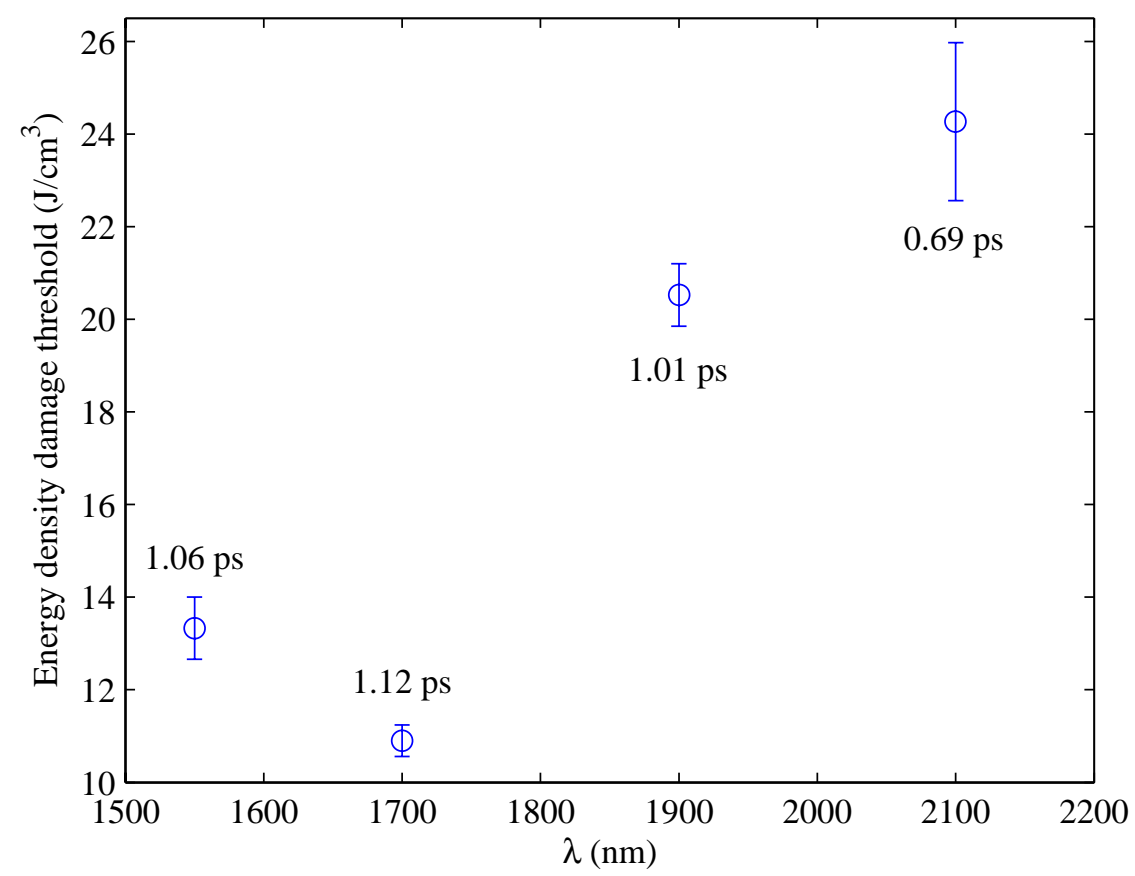

FIGURE 4. Damage thresholds for four wavelengths. The measured FWHM pulse width at each wavelength is shown next to the corresponding point.

damage process, and also because energy density can be related directly to accelerating gradient for a given structure geometry. Define the damage impedance for a structure by

$$
Z_{d}=\frac{E_{\mathrm{acc}}^{2}}{2 u_{\max } c},
$$

where $E_{\text {acc }}$ is the accelerating gradient on axis and $u_{\max }$ is the maximum electromagnetic energy density anywhere in the material. Then for a material with damage threshold $u_{\text {th }}$, the condition $u_{\max }<u_{\text {th }}$ sets an upper limit on $E_{\text {acc }}$. For the "woodpile" structure described in [5], we have $Z_{d}=6.84 \Omega$, yielding a maximum acceleration of $240 \mathrm{MeV} / \mathrm{m}$ at $\lambda=1550 \mathrm{~nm}$ from the data given above.

One single parameter is not guaranteed to give an accurate value for the maximum accelerating gradient in a structure, however. Because of the role of the electron avalanche and possibly tunnel ionization as well, the maximum value of $|\mathbf{E}|$ in the material may factor into the sustainable gradient in addition to $u_{\max }$. Because silicon has an indirect bandgap, the electromagnetic momentum density may also be important. Ultimately, tests of fabricated structures may reveal that detailed PIC simulations of the damage process are necessary to accurately predict the sustainable gradient value, since feature sizes are small and non-local effects may be important. 


\section{CONCLUSION AND FUTURE WORK}

We measured the damage threshold of silicon for picosecond pulses at several wavelengths. We found that silicon can withstand $13.3 \mathrm{~J} / \mathrm{cm}^{3}$ of energy density at $1550 \mathrm{~nm}$, which translates to $240 \mathrm{MeV} / \mathrm{m}$ acceleration in the woodpile structure. The damage threshold increases for longer wavelengths and shorter pulse widths. Thus silicon is still promising as an accelerator material; however, from the upper bound obtained at $2260 \mathrm{~nm}$, we found that $\mathrm{GeV} / \mathrm{m}$ accelerating gradients are unlikely in the near infrared.

We plan to refine this result by adjusting the OPA system to the same pulse duration for each wavelength. Also, we plan to collect complete data sets at wavelengths longer than the two-photon threshold.

\section{ACKNOWLEDGMENTS}

The author would like to thank R. Siemann, I. Blumenfeld, C. Clayton and M. Tang for helpful advice and R. Ischebeck and J. Spencer for the silicon sample, and extend special thanks to D. Simanovskii, G. Marcus, K. Cohn and D. Palanker of the Hansen Experimental Physics Laboratory at Stanford University for use of the laser system and assistance with the OPA. Work supported by Department of Energy contracts DE-AC0276SF00515 (SLAC) and DE-FG03-97ER41043-II (LEAP).

\section{REFERENCES}

1. D. F. Edwards, "Silicon," in Handbook of Optical Constants, edited by E. D. Palik, Academic Press, 1985, vol. 1, p. 547.

2. IMRA America, Inc., http : / / www . imra. com/.

3. K. M. Ho, C. T. Chan, C. M. Soukoulis, R. Biswas, and M. Sigalas, Solid State Commun. 89, 413-416 (1994).

4. E. Colby, G. Lum, T. Plettner, and J. Spencer, IEEE Trans. Nucl. Sci. 49, 2857-2867 (2002).

5. B. Cowan, "Photonic Crystal Laser-Driven Accelerator Structures," in Proceedings of the 2005 Particle Accelerator Conference, IEEE, Piscataway, NJ, 2005, pp. 731-733.

6. M. Lenzner, J. Krüger, S. Sartania, Z. Cheng, C. Spielmann, G. Mourou, W. Kautek, and F. Krausz, Phys. Rev. Lett. 80, 4076-4079 (1998).

7. B. C. Stuart, M. D. Feit, S. Herman, A. M. Rubenchik, B. W. Shore, and M. D. Perry, Phys. Rev. B 53, 1749-1761 (1996).

8. A. P. Joglekar, H. Liu, G. J. Spooner, E. Meyhöfer, G. Mourou, and A. J. Hunt, Appl. Phys. B 77, 25-30 (2003).

9. D. M. Simanovskii, H. A. Schwettman, H. Lee, and A. J. Welch, Phys. Rev. Lett. 91, 107601 (2003). 